\title{
THE STABILITY PROBLEM IN SHAPE, AND A WHITEHEAD THEOREM IN PRO-HOMOTOPY
}

\author{
BY \\ DAVID A. EDWARDS AND ROSS GEOGHEGAN(1)
}

\begin{abstract}
Theorem 3.1 is a Whitehead theorem in pro-homotopy for finite-dimensional pro-complexes. This is used to obtain necessary and sufficient algebraic conditions for a finite-dimensional tower of complexes to be pro-homotopy equivalent to a complex $(\S 4)$ and for a finite-dimensional compact metric space to be pointed shape equivalent to an absolute neighborhood retract ( $\$ 5)$.
\end{abstract}

1. Introduction. The theory of shape was introduced by Borsuk [2] for compact metric spaces (compacta) and was extended in a natural way by Fox [13] to all metric spaces. Shape theory agrees with homotopy theory on metric absolute neighborhood retracts (ANR's) and there is ample evidence that as a way of doing algebraic topology on "bad" spaces, shape theory is "better" than homotopy theory.

There is, of course, both shape theory and pointed shape theory. Borsuk [5] has shown that the distinction is important. What we call the stability problem (unpointed or pointed version) is loosely expressed by the question: when is a "bad" space shape equivalent to a "good" space? Specifically, for compacta, this takes two forms:

Problem A. Give necessary and sufficient conditions for a compactum $Z$ to have the Fox shape of an ANR.

Problem B. Give necessary and sufficient conditions for a compactum $Z$ to have the Borsuk shape of a compact polyhedron.

We lay the ground rules as follows. The conditions in Problems A and B should be intrinsic. Preferably they should be stated in terms of the algebraic shape invariants of $Z$.

When $Z$ is finite-dimensional and 1-UV, the second author and Lacher have given in [14] a satisfactory solution to Problem B, namely: a finite-dimensional 1.UV compactum $Z$ has the Borsuk shape of a compact polyhedron if and only if its Čech cohomology with integer coefficients is finitely generated. But without the hypothesis 1-UV, they have only given a necessary and sufficient embedding

Received by the editors September 30, 1974.

AMS (MOS) subject classifications (1970). Primary 55D99; Secondary 14 F99.

(1) The second-named author was supported in part by NSF Grant PO 38761. 
condition on $Z$, and by the present ground rules this is not acceptable.

We attempted to solve the pointed version of Problem $A$ in our paper [8], but we only found necessary and sufficient conditions for $Z$ to be weak shape equivalent to an ANR.

In the present paper we solve the pointed versions of Problems A and B for finite-dimensional compacta. For convenience we confine ourselves to connected compacta, but this is not a significant restriction. If $(Z, z)$ is a pointed compactum, let us denote the $k$ th homotopy pro-group of $(Z, z)$ by pro- $\pi_{k}(Z, z)$ and the (Borzuk) $k$ th shape group of $(Z, z)$ by $\pi_{k}(Z, z)$. See $\S \S 2$ and 5 for the definitions. The nature of our solution is best explained in the following abbreviated version of Theorem 5.1.

THEOREM 1.1. Let $(Z, z)$ be a pointed connected compact subset of some euclidean space. The following are equivalent:

(i) for each $k \geqslant 1$, pro- $\pi_{k}(Z, z)$ is isomorphic in the category pro-Groups to the group $\pi_{k}(Z, k)$;

(ii) $(Z, z)$ has the pointed Fox shape of a pointed ANR;

(iii) $(Z, z)$ is dominated in pointed Borsuk shape by a pointed compact polyhedron.

Furthermore, if $(Z, z)$ satisfies any of these conditions, there is an intrinsically defined "Wall obstruction" $w(Z, z)$ lying in the projective class group of $\underline{\pi}_{1}(Z, z)$ which vanishes if and only if $(Z, z)$ has the pointed Borsuk shape of a pointed compact polyhedron.

The Wall obstruction (so called because of its connection with [26]) mentioned in the above theorem does not always vanish. In fact

(1.2) we show how to construct pointed two-dimensional compacta which are dominated in pointed Borsuk shape by pointed compact polyhedra, but which do not have the pointed Borsuk shape of pointed compact polyhedra.

However, the Wall obstruction vanishes if $\pi_{1}(Z, z)$ is free or free abelian and so we have (see 5.2):

CoROllaRY 1.3. With $(Z, z)$ as above, suppose $\underline{\pi}_{1}(Z, z)$ is free or free abelian. Then $(Z, z)$ has the pointed Borsuk shape of a pointed compact polyhedron if and only if for each $k \geqslant 1$ pro- $\pi_{k}(Z, z)$ is isomorphic in the category pro-Groups to the group $\underline{\pi}_{k}(Z, z)$.

The condition that pro- $\pi_{k}(Z, z)$ be isomorphic to $\underline{\pi}_{k}(Z, z)$ looks forbidding, but it can often be checked. It is easier to check the equivalent condition (see 5.1) that $(Z, z)$ be movable and that the natural topology on $\pi_{k}(Z, z)$ be discrete. We give examples in [8] and we will not repeat them here.

As our title implies, we use a new Whitehead theorem in pro-homotopy 
theory, a sharpening of Whitehead theorems proved by Moszyńska [23] and Mardešić [20]. We do not improve their Whitehead theorems in shape theory, but by introducing a kind of mapping cylinder in pro-homotopy we are able to prove exactly the tool we need, Theorem 3.1. This theorem neither implies nor is implied by Mardešic's Whitehead theorem, but we use the key lemmas of his paper [20] in the proof. We also prove, as an easy corollary, Theorem 3.2 for towers (i.e., inverse systems indexed by the set of natural numbers) which more or less generalizes Theorem 3.7 of [23].

Our other theorem of interest in its own right is Theorem 4.2, in which we give necessary and sufficient conditions for a tower of complexes to be isomorphic in pro-homotopy to a complex. In fact our shape-theoretic results concerning compacta are merely corollaries of Theorem 4.2. We make the usual journey from compacta to towers of complexes and back to compacta.

It is natural that our solution to Problems A and B should be in pointed shape, because our algebraic condition concerns homotopy groups. By using a geometrical theorem of Siebenmann it is possible to give an unpointed version of Theorem 1.1. All reference to base points is dropped, except in part (i) where $z$ is an arbitrary point of $Z$; see [9].

In our paper [9] we study the "strange compacta" mentioned in (1.2). Their complements in euclidean spaces are very interesting open manifolds. In the same paper we give a different kind of solution to Problems A and B.

Theorem 1.1, and all the theorems in this paper, fail without a finite-dimensionality hypothesis. See $[18],[15],[1$, p. 35], [8], [7]. However, see also [10] .

In this paper we only consider Problems A and B for compacta. In [11] we will deal with other spaces.

2. Notation and terminology. This section is intended for reference.

If $C$ is a category there is category pro- $C$. The objects of pro- $C$ are inverse systems in $C$ indexed by directed sets. Thus pro- $C$ as defined here is a full subcategory of the category which Artin and Mazur call pro- $C$ [1, Appendix] , since they allow inverse systems to be indexed by "filtering categories", a notion more general than "directed set". We assume the reader is familiar" with ("our") pro-C. If not, he should consult [8] or [20]. When denoting an object of pro-C we suppress the bonding morphisms and the directed set which indexes the object; thus a typical object is denoted by $\left\{X_{\alpha}\right\}$, where $\alpha$ ranges over a directed set. If objects $\left\{X_{\alpha}\right\}$ and $\left\{Y_{\beta}\right\}$ appear as domain and range of a morphism it is understood that the indexing directed sets may differ, but if $\left\{X_{\alpha}\right\}$ and $\left\{Y_{\alpha}\right\}$ appear as domain and range, the indexing sets are understood to be the same.

An object of pro-C indexed by the directed set of natural numbers is called a tower in $C$. 
The following categories are used throughout: Groups (groups and homomorphisms); $T_{0}$ (pointed connected topological spaces and pointed maps); $H T_{0}$ (pointed connected topological spaces and homotopy classes of pointed maps relative to the base point $\left[25\right.$, p. 23]); $C W_{0}$ (pointed connected $C W$ complexes and pointed (not necessarily cellular) maps); $H_{0}$ (pointed connected $C W$ complexes and homotopy classes of pointed maps relative to the base point); $A N R_{0}$ (the full subcategory of $T_{0}$ generated by pointed ANR's); $H A N R_{0}$ (the full subcategory of $H T_{0}$ generated by pointed ANR's).

We think of a $C W$ complex as a space with additional structure, so that $C W_{0}$ [resp. $H_{0}$ ] is not a subcategory of $T_{0}$ [resp. $H T_{0}$ ]. When the context permits, we will identify $C W_{0}$ and $H_{0}$ with the corresponding subcategories of $T_{0}$ and $H T_{0}$.

We always suppress base points when describing objects of these categories of pointed spaces, similarly in the pro-categories. If $X=\left\{X_{\alpha}\right\}$ is an object of pro- $T_{0}$, pro- $H T_{0}$, etc. $\pi_{k}(X)$ will denote the object $\left\{\pi_{k}\left(X_{\alpha}\right)\right\}$ of pro-Groups, where $\pi_{k}\left(X_{\alpha}\right)$ is the $k$ th homotopy group of $X_{\alpha} . \check{\pi}_{k}(X)$ is the inverse limit group $\lim _{\alpha}\left\{\pi_{k}\left(X_{\alpha}\right)\right\}$. A morphism of pro-CW or pro- $H_{0}$ is a weak equivalence if it induces an isomorphism on $\pi_{k}$ for all $k \geqslant 1$.

If $\left\{X_{\alpha}\right\}$ is in pro- $T_{0}$ [resp. pro-CW $W_{0}$ we also denote by $\left\{X_{\alpha}\right\}$ the corresponding object of pro- $H T_{0}$ [resp. pro- $H_{0}$ ].

The $C W$ dimension of a $C W$ complex $X_{\alpha}$ is the integer $C W$-dim $X_{\alpha}$ such that $X_{\alpha}$ contains cells of that dimension but of no higher dimension. If no such integer exists, $C W-\operatorname{dim} X_{\alpha}=\infty$. If $X=\left\{X_{\alpha}\right\}$ is an object of pro-CW or pro- $H_{0}$, $C W$-dim $X$ is defined to be $\sup _{\alpha}\left\{C W-\operatorname{dim} X_{\alpha}\right\}$. On the other hand the homotopy dimension of $X$ is

$h-\operatorname{dim} X=\inf \left\{C W-\operatorname{dim} Y \mid Y\right.$ is isomorphic to $X$ in pro- $\left.H_{0}\right\}$.

Clearly $h$-dim $X \leqslant C W$-dim $X$.

An object $X=\left\{X_{\alpha}\right\}$ of pro-CW is compact if each $X_{\alpha}$ is a finite complex.

If $G$ is a group, its projective class group (see for example [26, p. 64]) is denoted by $\widetilde{K}^{0}(G)$.

Other notation and terminology will be introduced as required.

3. Whitehead theorems in pro-homotopy. Our principal Whitehead theorem is

THEOREM 3.1 (Whitehead theorem in pro-CW $W_{0}$ ). Let $g: X \rightarrow Y$ be a morphism of pro- $C W_{0}$ and let

$$
n=\max \{1+C W-\operatorname{dim} X, C W-\operatorname{dim} Y\}<\infty .
$$

Suppose $g_{\#}: \pi_{k}(X) \rightarrow \pi_{k}(Y)$ is an isomorphism (in the category pro-Groups) for 
$k \leqslant n$ and suppose $g_{\#}$ has a right inverse for $k=n+1$. Then $g$ induces an isomorphism of pro- $H_{0}$.

From this, we easily derive

THEOREM 3.2 (Whitehead theorem for towers). Let $X$ and $Y$ be towers in $H_{0}$; let $g: X \rightarrow Y$ be a morphism of pro- $H_{0}$ and let

$$
n=\max \{1+C W-\operatorname{dim} X, C W-\operatorname{dim} Y\}<\infty .
$$

Suppose $g_{\#}: \pi_{k}(X) \rightarrow \pi_{k}(Y)$ is an isomorphism (in the category pro-Groups) for $k \leqslant n$ and suppose $g_{\#}$ has a right inverse for $k=n+1$. Then $g$ is an isomorphism.

Before proving these theorems, we make some remarks.

(i) Artin and Mazur [1 p. 35] show that without the hypothesis $n<\infty$, $g$ is a -isomorphism (i.e., $g$ induces an isomorphism of Postnikov systems in pro- $H_{0}$ ), but that $g$ need not itself be an isomorphism in pro- $H_{0}$. We will use the fact that $g$ is a -isomorphism in the proof of Theorem 4.2.

(ii) In $3.2 \mathrm{~g}$ is in pro- $H_{0}$, while in $3.1 \mathrm{~g}$ is in pro-CW . We can only define mapping cylinders for morphisms in pro- $C W_{0}$. We know how to "lift" a morphism from pro- $H_{0}$ to pro- $C W_{0}^{\prime}$ only if the domain and range are towers.

(iii) The reader familiar with [23] and [20] will note that we avoid kernels, cokernels, epimorphisms, and bimorphisms in pro-Groups. Lemma 3.6 makes them unnecessary.

The proofs of Theorems 3.1 and 3.2 are at the end of this section following a series of lemmas.

Let $C$ be a category. $C_{\text {maps }}$ is the category whose objects are the morphisms of $C$ and whose morphisms between objects $f$ and $g$ are the commutative square diagrams

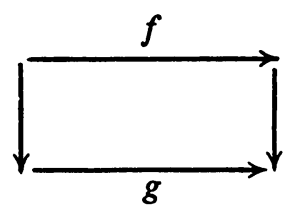

in $C$. Similarly, $C_{\Delta}$ is the category whose objects are commutative triangles in $C$ and whose morphisms are commutative prisms in $C$. There is an obvious functor

On objects,

$$
F: \text { pro- }\left(C_{\text {maps }}\right) \rightarrow(\text { pro- } C)_{\text {maps }} \text {. }
$$

$$
F \text { sends }\left\{X_{\alpha} \stackrel{f_{\alpha}}{\longrightarrow} Y_{\alpha}\right\} \text { to }\left\{X_{\alpha}\right\} \stackrel{\left\{f_{\alpha}\right\}}{\longrightarrow}\left\{Y_{\alpha}\right\}
$$

where $\left\{f_{\alpha}\right\}$ stands for the morphism of pro- $C$ generated by the maps $f_{\alpha}$.

We will need to replace objects of (pro-C) maps by "equivalent" objects of 
pro- $\left(C_{\text {maps }}\right)$. This is achieved by

Lemma 3.3. Let $g:\left\{X_{\alpha}\right\} \rightarrow\left\{Y_{\beta}\right\}$ be an object of (pro-C $)_{\text {maps }}$. Then there exists an object

$$
f=\left\{X_{\gamma}^{\prime} \stackrel{f_{\gamma}}{\longrightarrow} Y_{\gamma}^{\prime}\right\}
$$

of pro- $\left(C_{\text {maps }}\right)$ such that $F(f)$ is isomorphic to $g$, each $X_{\gamma}^{\prime}$ is some $X_{\alpha}$ and each $Y_{\gamma}^{\prime}$ is some $Y_{\beta}$.

This is proved in $[20, \S 2.2]$. Compare $[1$, p. 160].

A directed set is closure finite if each element has only finitely many predecessors. We may always work with closure finite indexing sets (up to isomorphism) because of

LEMMA 3.4. Let $\left\{X_{\alpha}\right\}$ be an object of pro-C. Then there exists an object $\left\{X_{\beta}^{\prime}\right\}$ of pro-C, indexed by a closure finite directed set, such that $\left\{X_{\beta}^{\prime}\right\}$ is isomorphic to $\left\{X_{\alpha}\right\}$ and each $X_{\beta}^{\prime}$ is some $X_{\alpha}$.

This is proved in $[20, \S 2.3]$.

The next "replacement lemma" is obvious.

LEMMA 3.5. Let $\left\{X_{\alpha}\right\}$ be an object of pro-C indexed by a directed set $A$. For each $\alpha \in A$, let $e_{\alpha}: X_{\alpha} \rightarrow X_{\alpha}^{\prime}$ be an isomorphism in $C$, and for each $\alpha \leqslant \beta$ $\in A$ let $f_{\alpha \beta}: X_{\beta} \rightarrow X_{\alpha}$ be the appropriate bonding morphism of $\left\{X_{\alpha}\right\}$. Then the object $\left\{X_{\alpha}^{\prime}\right\}$ with bonding morphisms $e_{\alpha} \circ f_{\alpha \beta} \circ e_{\beta}^{-1}: X_{\beta}^{\prime} \rightarrow X_{\alpha}^{\prime}$ is isomorphic in pro-C to $\left\{X_{\alpha}\right\}$.

If $f: X \rightarrow Y$ is a morphism of $T_{0}$, the "relative homotopy group" $\pi_{k}(f)$ satisfies the usual long exact sequence $[16$, p. 21$]$. If $f$ is an inclusion, $\pi_{k}(f)$ is functorially isomorphic to the usual $\pi_{k}(Y, X)$. Note. To avoid ambiguity $f_{\#}$, not $\pi_{k}(f)$, will be used for the homomorphism $\pi_{k}(X) \rightarrow \pi_{k}(Y)$ induced by $f$.

LEMMA 3.6. Let

$$
f=\left\{X_{\alpha} \stackrel{f_{\alpha}}{\longrightarrow} Y_{\alpha}\right\}
$$

be an object of pro- $\left(T_{0, \text { maps }}\right)$, let $k \geqslant 1$ be an integer, and let

$$
\left\{\pi_{k}\left(X_{\alpha}\right) \stackrel{f_{\alpha \#}}{\longrightarrow} \pi_{k}\left(Y_{\alpha}\right)\right\}
$$

have a right inverse and

$$
\left\{\pi_{k-1}\left(X_{\alpha}\right) \stackrel{f_{\alpha \#}}{\longrightarrow} \pi_{k-1}\left(Y_{\alpha}\right)\right\}
$$

have a left inverse in pro-Groups. Then $\pi_{k}(f) \equiv\left\{\pi_{k}\left(f_{\alpha}\right)\right\}$ is trivial (in proSets if $k=1$, in pro-Groups otherwise). 
Proof. Given $\alpha$ there exist $\beta \geqslant \alpha$ and $\gamma \geqslant \beta$ and homomorphisms $a$ and $b$ such that the following diagram commutes.

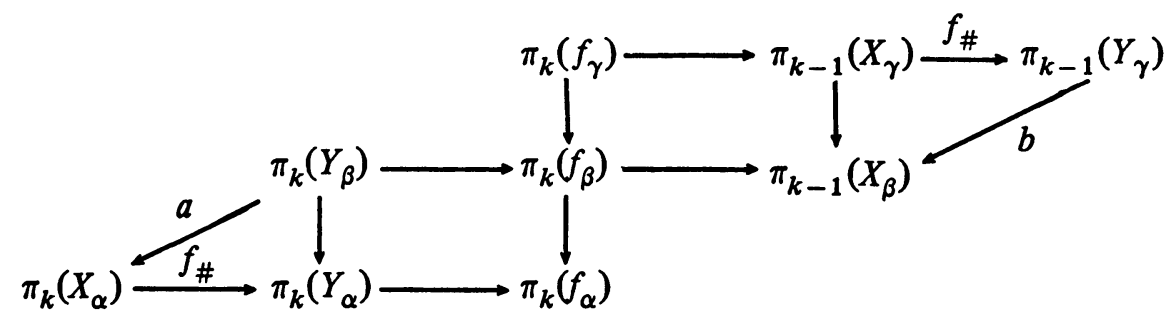

In this diagram the horizontal lines come from the ordinary homotopy exact sequences. By a diagram chase one easily sees that the homomorphism $\pi_{k}\left(f_{\gamma}\right)$ $\rightarrow \pi_{k}\left(f_{\alpha}\right)$ is zero. Hence $\pi_{k}(f)$ is trivial.

Let $f_{\alpha}: X_{\alpha} \rightarrow Y_{\alpha}$ be a morphism of $T_{0}$. Then the following diagram commutes in $T_{0}$.

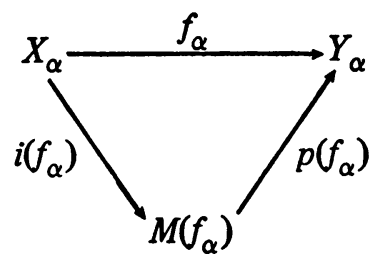

Here $M\left(f_{\alpha}\right)$ denotes the reduced mapping cylinder of $f_{\alpha}, i\left(f_{\alpha}\right)$ is the natural inclusion, and $p\left(f_{\alpha}\right)$ is the natural projection map. $p\left(f_{\alpha}\right)$ is a pointed homotopy equivalence. (See $[20, \S 7.1]$ for precise definitions; for $g$ there, read $p$ here).

Now suppose

$$
f=\left\{X_{\alpha} \stackrel{f_{\alpha}}{\longrightarrow} Y_{\alpha}\right\}
$$

is an object of pro-( $\left(T_{0, \text { maps }}\right)$. Since $M$ is a functor, and $i$ and $p$ are natural transformations, the diagrams (*) fit together to form an object of pro- $\left(T_{0, \Delta}\right)$ and

$$
p(f)=\left\{M\left(f_{\alpha}\right) \stackrel{p\left(f_{\alpha}\right)}{\longrightarrow} Y_{\alpha}\right\}
$$

and

$$
i(f)=\left\{X_{\alpha} \stackrel{i\left(f_{\alpha}\right)}{\longrightarrow} M\left(f_{\alpha}\right)\right\}
$$

are objects of pro- $\left(T_{0, \text { maps }}\right)$. Since each $p\left(f_{\alpha}\right)$ is a pointed homotopy equivalence, $F(p(f))$ induces an isomorphism of pro-HT $T_{0}$. Hence we have proved

LEMMA 3.7. $F(i(f))$ induces an isomorphism in pro-HT $T_{0}$ if and only if $F(f)$ does.

The diagram (*) leads to the following commutative diagram, $k \geqslant 1$. 


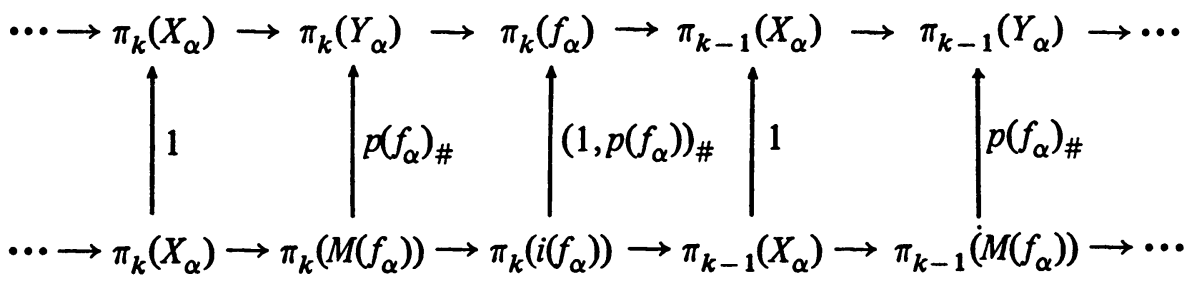

By the Five Lemma, $\left(1, p\left(f_{\alpha}\right)\right)_{\#}$ is an isomorphism. The construction is functorial, so $\pi_{k}(f) \equiv\left\{\pi_{k}\left(f_{\alpha}\right)\right\}$ and $\pi_{k}(i(f)) \equiv\left\{\pi_{k}\left(i\left(f_{\alpha}\right)\right)\right\}$ are isomorphic. Hence Lemma 3.6 implies

LEMMA 3.8. If $f$ satisfies the hypotheses of Lemma $3.6, \pi_{k}(i(f))$ is trivial. In other words $\left\{\pi_{k}\left(M\left(f_{\alpha}\right), X_{\alpha}\right)\right\}$ is trivial.

Let $H T_{0 \text {, pairs }}$ be the category whose objects are pointed pairs of spaces and whose morphisms are homotopy classes of pointed maps relative to the base point [25, p. 23]. Let $H_{0 \text {, pairs }}$ be the corresponding category generated by (pointed) pairs $\left(X, X^{\prime}\right)$ such that $X$ is a $C W$ complex and $X^{\prime}$ is a subcomplex. There is an obvious functor $G: H T_{0 \text {, pairs }} \rightarrow H T_{0 \text {, maps }}$ : on objects $G$ sends $\left(X, X^{\prime}\right)$ to the "inclusion" $X^{\prime} \rightarrow X$. There is a similar functor $G: H_{0 \text {, pairs }} \rightarrow H_{0 \text {, maps }}$. Both functors extend to pro-categories.

In the preceding three lemmas, $f$ has been in pro- $\left(T_{0, \text { maps }}\right)$, though in proving the Whitehead theorem our $f$ will be in the category pro-( $\left(C W_{0, \text { maps }}\right)$. But, even when $f$ is in pro- $\left(C W_{0, \text { maps }}\right),\left\{\left(M\left(f_{\alpha}\right), X_{\alpha}\right)\right\}$ only get us into pro-HT , pairs, not into pro- $H_{0 \text { pairs }}$ (we are not requiring the $f_{\alpha}$ 's to be cellular).

We will want to replace $\left\{\left(M\left(f_{\alpha}\right), X_{\alpha}\right)\right\}$ by an object $\left\{\left(P_{\alpha}, P_{\alpha}^{\prime}\right)\right\}$ in pro- $H_{0}$, pairs in which each $\left(P_{\alpha}, P_{\alpha}^{\prime}\right)$ is simplicial and finite dimensional. The next three trivial and well-known lemmas (3.9-3.11) achieve this. They are proved because we lack references.

Lemma 3.9. Let $f, g: X \rightarrow Y$ be morphisms of $T_{0}$ which are homotopic relative to the base point. Then $(M(f), X)$ and $(M(g), X)$ are isomorphic in $H T_{\text {0, pairs }}$.

Proof. (Notation as in $[20, \S 7]$.) Let $H: X \times I \rightarrow Y$ satisfy $H(\cdot, 0)=$ $g, H(\cdot, 1)=f$ and $H$ (basepoint $\times I)=$ basepoint. Define $H^{\prime}: X \times I \rightarrow Y$ by $H^{\prime}(x, t)=H(x, 1-t)$. Define $k:(M(f), X) \rightarrow(M(g), X)$ by

$$
\begin{aligned}
k([x, t]) & =[x, 2 t], & & 0 \leqslant t \leqslant 1 / 2, x \in X, \\
k([x, t]) & =[H(x, 2 t-1)], & & 1 / 2 \leqslant t \leqslant 1, x \in X, \\
k([y]) & =[y], & & y \in Y,
\end{aligned}
$$

and define $k^{\prime}:(M(g), X) \rightarrow(M(f), X)$ by the similar formula in which $k^{\prime}$ replaces 


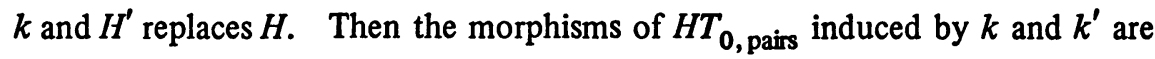
easily seen to be mutually inverse.

Corollary 3.10. If $f: X \rightarrow Y$ is a morphism of $C W_{0}$ then $(M(f), X)$ is isomorphic in $H T_{0 \text {, pairs }}$ to an object $(M(g), X)$ of $H_{0 \text {,pairs }}$ where $C W$-dim $M(g)=$ $\max (1+C W$-dim $X, C W$-dim $Y)$.

Proof. By the cellular approximation theorem, $f$ is homotopic to a cellular map $g .(M(g), X)$ is a $C W$ pair $[19$, p. 62] of the appropriate dimension. Apply Lemma 3.9.

We must replace $C W$ complexes by simplicial complexes of the same dimension, so the usual "singular complex" method is not appropriate. The following proof "by hand" is well known.

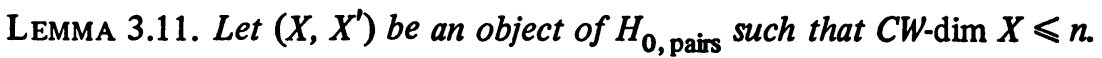
Then there exists a pointed pair of simplicial complexes $\left(Y, Y^{\prime}\right)$ such that $C W$-dim $Y \leqslant n$, and $\left(X, X^{\prime}\right)$ is isomorphic to $\left(Y, Y^{\prime}\right)$ in $H_{0 \text {, pairs }}$

Proof. We sketch the absolute case $X^{\prime}=\varnothing$; obvious modifications give the relative case. The proof is by induction on $n$ : The case $n=0$ is trivial. Let $X^{n-1}$ be the $(n-1)$-skeleton of the $n$-dimensional complex $X$. Assume there is an $(n-1)$-dimensional simplicial complex $Y^{n-1}$ and a homotopy equivalence $h$ : $X^{n-1} \rightarrow Y^{n-1}$. Let $X$ be obtained from $X^{n-1}$ by the attaching map $f$ : $\bigcup_{\lambda}\left\{\Delta_{\lambda}^{n}\right\} \rightarrow X^{n-1}$ where $\bigcup_{\lambda}\left\{\Delta_{\lambda}^{n}\right\}$ is a disjoint collection of "standard" $n$-simplexes, one for each $n$-cell of $X$. For a suitable subdivision $K$ of $\bigcup_{\lambda}\left\{\Delta_{\lambda}^{n}\right\}$, let $g: K \rightarrow Y_{n-1}$ be a simplicial approximation to $h \circ f$. Then $h$ extends to a homotopy equivalence from $X$ to the $n$-dimensional $C W$ complex

$$
\tilde{Y} \equiv Y_{n-1} \cup_{g}\left(\bigcup_{\lambda}\left\{\Delta_{\lambda}^{n}\right\}\right)
$$

$[19$, p. 122]. It is well known that if $L$ is the second barycentric subdivision of $\cup_{\lambda}\left\{\Delta_{\lambda}^{n}\right\}$ relative to $K$ (i.e. simplexes of $K$ are not starred) $L$ induces a simplicial subdivision $Y$ of $\tilde{Y}$ such that $Y_{n-1}^{n}$ is a subcomplex of $Y$.

The following is essentially Theorem 2 of [20].

Proposition 3.12. Let $\widetilde{P} \equiv\left\{\left(P_{\alpha}, P_{\alpha}^{\prime}\right)\right\}$ be an object of pro- $\left(H_{0, \text { pairs }}\right)$ indexed by a closure finite directed set. Assume that each $P_{\alpha}$ is a simplicial complex of dimension $\leqslant n<\infty$ and that $P_{\alpha}^{\prime}$ is a subcomplex of $P_{\alpha}$. If $\left\{\pi_{k}\left(P_{\alpha}, P_{\alpha}^{\prime}\right)\right\}$ is trivial for $1 \leqslant k \leqslant n+1$, then the "inclusion" $F \cdot G(\widetilde{P}):\left\{P_{\alpha}^{\prime}\right\} \rightarrow\left\{P_{\alpha}\right\}$ is an isomorphism in pro- $H_{0}$.

Proof OF THEOREM 3.1. Lemmas 3.3 and 3.4 imply the existence of an object

$$
f=\left\{X_{\gamma}^{\prime} \stackrel{f_{\gamma}}{\longrightarrow} Y_{\gamma}^{\prime}\right\}
$$


of pro- $\left(C W_{0, \text { maps }}\right)$ indexed by a closure finite directed set, such that $n=$ $\max \left\{1+C W-\operatorname{dim}\left\{X_{\gamma}^{\prime}\right\}, C W-\operatorname{dim}\left\{Y_{\gamma}^{\prime}\right\}\right\}$, and $F(f)$ is isomorphic to $g$ in (pro-CW $\left.)_{0}\right)_{\text {maps }}$. Hence $f_{\#}:\left\{\pi_{k}\left(X_{\gamma}^{\prime}\right)\right\} \rightarrow\left\{\pi_{k}\left(Y_{\gamma}^{\prime}\right)\right\}$ is an isomorphism in the category pro-Groups for $k \leqslant n, f_{\#}$ has a right inverse for $k=n+1$, and it is enough to show that $F(f)$ induces an isomorphism of pro- $H_{0}$. By Lemma $3.8,\left\{\pi_{k}\left(M\left(f_{\gamma}\right), X_{\gamma}^{\prime}\right)\right\}$. is trivial, $1 \leqslant k \leqslant n+1$, and by Lemma 3.7 we need only show that the "inclusion" $F(i(f)):\left\{X_{\gamma}^{\prime}\right\} \rightarrow\left\{M\left(f_{\gamma}\right)\right\}$ induces an isomorphism of pro-HT . By 3.10, 3.11 and $3.5\left\{\left(M\left(f_{\gamma}\right), X_{\gamma}^{\prime}\right)\right\}$ is isomorphic in pro- $\left(H T_{0, \text { pairs }}\right)$ to an object $\left\{\left(P_{\gamma}, P_{\gamma}^{\prime}\right)\right\}$ where each $\boldsymbol{P}_{\boldsymbol{\gamma}}$ is a simplicial complex of dimension $\leqslant n, P_{\gamma}^{\prime}$ is a subcomplex of $P_{\gamma}$, and $\left\{\pi_{k}\left(P_{\gamma}, P_{\gamma}^{\prime}\right)\right\}$ is trivial for $1 \leqslant k \leqslant n+1$. By Proposition 3.12 the "inclusion"

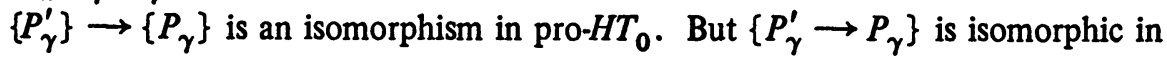
pro- $\left(H T_{0, \text { maps }}\right)$ to

$$
\left\{X_{\gamma}^{\prime} \stackrel{i\left(f_{\gamma}\right)}{\longrightarrow} M\left(f_{\gamma}\right)\right\}
$$

(applying the functor $G$ ). Hence the induced "inclusion" $\left\{P_{\gamma}^{\prime}\right\} \rightarrow\left\{P_{\gamma}\right\}$ is isomorphic in (pro- $\left.H T_{0}\right)_{\text {maps }}$ to the "inclusion" $\left\{X_{\gamma}^{\prime}\right\} \rightarrow\left\{M\left(f_{\gamma}\right)\right\}$. Since the former is an isomorphism in pro- $H T_{0}$, and the latter is the morphism of pro- $H_{0}$ induced by $F(i(f))$, the theorem is proved.

Proof of Theorem 3.2. We reduce 3.2 to 3.1 in much the same way as Mardešic reduces 7.2 to 6.5 in [20]. By reindexing $Y$ we may assume without loss of generality that $g=F(f)$ where $f=\left\{f_{n}: X_{n} \rightarrow Y_{n}\right\}$ is an object of pro$\left(H_{0, \text { maps }}\right)$. This is done "by hand" and does not require Lemma 3.3. Let $a_{n}: X_{n+1} \rightarrow X_{n}$ and $b_{n}: Y_{n+1} \rightarrow Y_{n}$ be cellular maps representing the bonding homotopy classes of $X$ and $Y$, and let $\varphi_{n}: X_{n} \rightarrow Y_{n}$ be a cellular map representing the homotopy class $f_{n}$. Then the diagram in $C W_{0}$

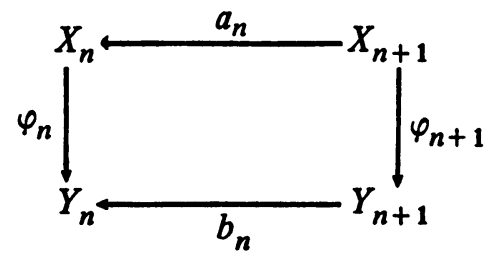

induces a commutative diagram in $H_{0}$. From it we obtain a commutative diagram in $C W_{0}$

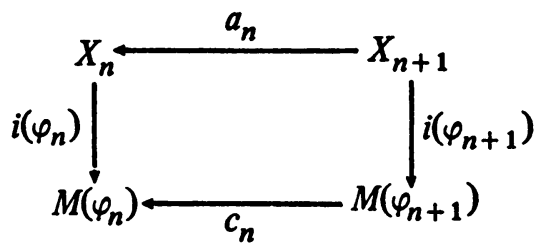

as follows. Let $H_{n+1}: X_{n+1} \times I \rightarrow Y_{n}$ be a cellular homotopy between $\varphi_{n} \circ a_{n}$ (on level 0 ) and $b_{n} \circ \varphi_{n+1}$ (on level 1), such that $H_{n+1}$ (basepoint $\left.\times I\right)=$ 
basepoint; $c_{n}$ is defined by

$$
\begin{array}{rlrl}
c_{n}([x, t]) & =[x, 2 t], & & 0 \leqslant t \leqslant 1 / 2, x \in X_{n+1}, \\
c_{n}([x, t]) & =\left[H_{n+1}(x, 2 t-1)\right], & 1 / 2 \leqslant t \leqslant 1, x \in X_{n+1}, \\
c_{n}([y]) & =\left[b_{n}(y)\right], & & y \in Y_{n+1} .
\end{array}
$$

The diagrams

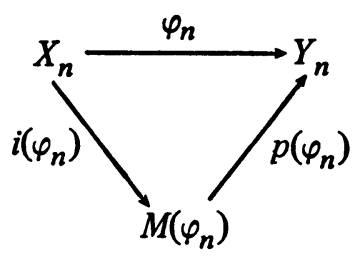

commute in $C W_{0}$ and fit together to form an object of pro- $\left(H_{0, \Delta}\right)$ bonded by the homotopy classes of $a_{n}, b_{n}$ and $c_{n}$. The resulting commutative triangle in pro- $H_{0}$ is

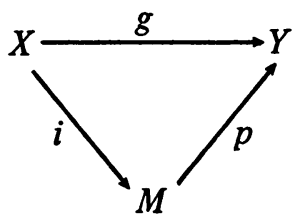

where $p$ is an isomorphism. Since $i$ is induced by the morphism $\left\{i\left(\varphi_{n}\right)\right\}$ of pro$C W_{0}$, and the latter satisfies the hypotheses of Theorem 3.1, $i$ is an isomorphism in pro- $H_{0}$. Hence $g$ is an isomorphism in pro- $H_{0}$.

Concluding remark. In the rest of this paper we will deal only with towers, and so we will use only Theorem 3.2. A version of Theorem 3.1 will be used in [11]. A (limited) infinite-dimensional version of Theorem 3.1 will be given in [10].

4. Criteria for a tower of complexes to be pro-homotopy equivalent to a complex and to a finite complex.

LEMMA 4.1. Let $\left\{G_{\alpha}\right\}$ be an object of pro-Groups, let $G$ be its inverse limit and let $p: G \rightarrow\left\{G_{\alpha}\right\}$ be the projection morphism. If $\left\{G_{\alpha}\right\}$ is isomorphic to a group, then $p$ is an isomorphism (in pro-Groups).

Proof. Let $\left\{G_{\alpha}\right\}$ be isomorphic to the group $H$. Let the isomorphism be $\varphi$ generated by $\varphi_{\beta}: G_{\beta} \rightarrow H$, and let its inverse be $\psi$, generated by $\left\{\psi_{\alpha}: H \rightarrow\right.$ $G_{\alpha}$ \}. It will be enough to show that $\varphi \circ p: G \rightarrow H$ is an isomorphism of groups. $\varphi \circ p$ is surjective, for if $h \in H$, the element $\left(\psi_{\alpha}(h)\right)$ of $G$ is mapped to $h$ by $\varphi \circ p$. $\varphi \circ p$ is injective, for suppose $\left(g_{\alpha}\right)$ lies in the kernel of $\varphi \circ p$; then for any $\alpha$, there exists $\gamma \geqslant \alpha, \beta$ such that $\psi_{\alpha} \circ \varphi_{\beta} \circ$ bond $\left(g_{\gamma}\right)=e=g_{\alpha}$, so $\left(g_{\alpha}\right)$ is the identity element of $G$.

THEOREM 4.2 (stability theorem for towers). Let $X=\left\{X_{n}\right\}$ be a tower 
in $H_{0}$. (i) There exist a pointed $C W$ complex $Q$ and a weak equivalence $q: Q \rightarrow X$ in pro- $H_{0}$ if and only if $\pi_{k}(X)$ is isomorphic in pro-Groups to the group $\check{\pi}_{k}(X)$ for all $k \geqslant 1$. In case the condition in (i) holds we have

(ii) $Q$ may be chosen so that $C W-\operatorname{dim} Q=\max \{3, h-\operatorname{dim} X\}$ and if $h-\operatorname{dim} X=$ $1, Q$ may be chosen to be a bouquet of circles;

(iii) if $C W$-dim $X<\infty, q$ is an isomorphism in pro- $H_{0}$;

(iv) if $C W$ - $\operatorname{dim} X<\infty$ and $X$ is compact, then $Q$ is dominated (in $H_{0}$ ) by a finite complex: in which case there is an intrinsically defined "Wall obstruction" $w(X) \in \widetilde{K}^{0}\left(\check{\pi}_{1}(X)\right)$ which vanishes if $X$ is isomorphic in pro- $H_{0}$ to a finite complex, and whose vanishing implies that $X$ is isomorphic in pro- $H_{0}$ to a finite complex of dimension $\max \{3, h$-dim $X\}$;

(v) all possible Wall obstructions occur among towers $X$ such that $C W$-dim $X=2$.

We remark that (v) is nonvacuous. There are groups $G$ for which $\widetilde{K}^{0}(G)$ is nontrivial; for example the cyclic group of order 23 (see [24, p. 711]). On the other hand $\widetilde{K}^{0}(G)$ is often trivial, for example if $G$ is free or free abelian. So if $X$ is a finite-dimensional compact tower and $\check{\pi}_{1}(X)$ is suitable, Theorem 4.2 says that $X$ is isomorphic to a finite complex if and only if $\pi_{k}(X)$ is isomorphic to $\check{\pi}_{k}(X)$ for all $k \geqslant 1$.

Proof of (i). First we prove "only if". The following diagram obviously commutes in pro-Groups.

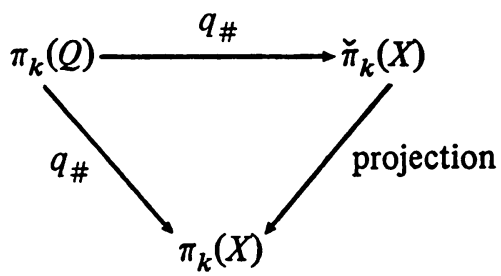

We are given that the slanting morphism $q_{\#}$ is an isomorphism. Since lim: proGroups $\rightarrow$ Groups is a functor, it follows that the horizontal morphism $q_{\#}$ is an isomorphism in Groups, a fortiori in pro-Groups; this could also be proved directly. Hence "projection" is an isomorphism in pro-Groups.

"If" is essentially proved in our paper [8], so we will merely sketch the proof here. Let $K_{0}$ be the category of pointed connected Kan complexes and pointed simplicial maps [22]. Let $Y \equiv\left\{Y_{n}\right\}$ be a tower in $K_{0}$ such that every bonding morphism $Y_{n+1} \rightarrow Y_{n}$ is a Kan fibration [22], and let $\bar{Y}$ be the inverse limit of $Y . \bar{Y}$ is a pointed Kan complex. It is proved in [6, IX. 3.1] that the natural projection morphism $p: \bar{Y} \rightarrow Y$ gives the following exact sequence of groups for each $k \geqslant 1$ :

$$
\text { (point) } \rightarrow \lim _{n}^{1}\left\{\pi_{k+1}\left(Y_{n}\right)\right\} \rightarrow \pi_{k}(\bar{Y}) \stackrel{p_{\#}}{\rightarrow} \check{\pi}_{k}(Y) \rightarrow \text { (point). }
$$


If $\left\{\pi_{k+1}\left(Y_{n}\right)\right\}$ is isomorphic to a group, $\lim _{n}^{1}\left\{\pi_{k+1}\left(Y_{n}\right)\right\}$ is trivial (this is easily proved directly, though it also follows from [6. p. 256, Corollary 3.5]); thus $p_{\#}$ is an isomorphism of groups for $k \geqslant 1$, and $\bar{Y}$ is connected. If $\pi_{k}(Y)$ is isomorphic in pro-Groups to $\check{\pi}_{k}(Y)$, Lemma 4.1 implies that the corresponding $p_{\#}: \pi_{k}(\bar{Y}) \rightarrow$ $\left\{\pi_{k}\left(Y_{n}\right)\right\}$ is an isomorphism in pro-Groups, since the triangle analogous to (**) commutes.

Let $H K_{0}$ be the homotopy category corresponding to $K_{0}$. Let $S$ and $|\cdot|$ be the singular-complex and geometric-realization functors [22].

We apply the above remarks. There exist a tower of fibrations $\left\{Y_{n}\right\}$ and a morphism of pro- $K_{0},\left\{S\left(X_{n}\right)\right\} \rightarrow\left\{Y_{n}\right\}$, which induces an isomorphism in pro$H K_{0}$. Hence we have a composition in pro- $H_{0}$ :

$$
q: Q \equiv|\bar{Y}| \stackrel{|p|}{\longrightarrow}\left\{\left|Y_{n}\right|\right\} \rightarrow\left\{\left|S\left(X_{n}\right)\right|\right\} \rightarrow\left\{X_{n}\right\} \equiv X
$$

where the unmarked arrows are isomorphisms in pro- $H_{0}$. The hypothesis implies that for each $k \geqslant 1, \pi_{k+1}(Y) \equiv\left\{\pi_{k+1}\left(Y_{n}\right)\right\}$ is isomorphic to a group and that $\pi_{k}(Y)$ is isomorphic to $\breve{\pi}_{k}(Y)$; hence $p$ is a weak equivalence in pro- $K_{0}$. Therefore $q$ is a weak equivalence.

Proof of (ii). By Corollary 4.4 of [1], the weak equivalence $q: Q \rightarrow X$ induces an isomorphism in pro- $H_{0} q^{\natural}: Q^{\natural} \rightarrow X^{\natural}$ (of the associated Postnikov systems). Hence, by 4.4 (ii) of [1] $q$ induces isomorphisms of homology progroups and of cohomology groups (with every possible coefficient bundle). But the canonical morphisms $Q \rightarrow Q^{\natural}$ and $X \rightarrow X^{\natural}$ of pro- $H_{0}$ clearly induce isomorphisms of homology and cohomology. Since the square

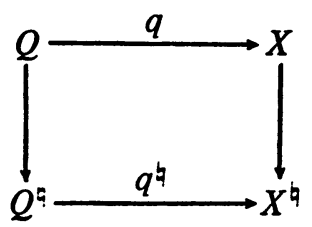

commutes, $q$ must induce isomorphisms of homology and cohomology. Hence, in particular, the cohomology of $Q$ vanishes in dimensions above $h$-dim $X$. If we let $\widetilde{X}=\left\{\widetilde{X}_{n}\right\}$, where $\widetilde{X}_{n}$ is the (pointed) universal cover of $X_{n}$ and the bonding morphisms of $\widetilde{X}$ are (uniquely) lifted from those of $X$, we have a weak equivalence $\widetilde{q}: \widetilde{Q} \rightarrow \widetilde{X} . h-\operatorname{dim} \widetilde{X} \leqslant h-\operatorname{dim} X$, because $X$ is isomorphic to some $X^{\prime}$ in pro- $H_{0}$ such that $h-\operatorname{dim} X=C W$-dim $X^{\prime}=C W$-dim $\widetilde{X}^{\prime} \geqslant h-\operatorname{dim} \widetilde{X}$. So the above argument shows that the homology of $\widetilde{Q}$ vanishes in dimensions above $h-\operatorname{dim} X$. Wall has shown that if the cohomology of $Q$ and the homology of $\widetilde{Q}$ vanish above dimension $d$, then $Q$ is homotopy equivalent to a complex of dimension $\max \{3, d\}$, and that $Q$ is homotopy equivalent to a bouquet of circles if $d=1$ [26, Theorems D and E]. So (ii) is proved.

Proof of (iii). Combine (i), (ii), and the Whitehead Theorem 3.2. 
Proof of (iv). Let the topological inverse limit of $X$ be $Z . Z$ is a (pointed) compact metric space. The set pro- $H_{0}(X, Q)$ of morphisms of pro- $H_{0}$ from $X$ to $Q$ can be identified with $\lim _{n}\left[X_{n}, Q\right]$, where $[A, B]$ denotes the set of morphisms of $H T_{0}$ from $A$ to $\vec{B}$ (see [1, p. 154]). Hence there is a natural bijection $\lambda_{Q}:$ pro- $H_{0}(X, Q) \rightarrow[Z, Q]$ (see [12, p. 287] or [25, p. 152]). By (ii), $q:$ $Q \rightarrow X$ induces an isomorphism (also denoted by $q$ ) in pro- $H_{0}$. Let its inverse in pro- $H_{0}$ be $q^{-1}: X \rightarrow Q$, and let $r: Z \rightarrow \mathcal{C}$ be a morphism of $T_{0}$ whose homotopy class corresponds to $q^{-1}$ under $\lambda_{Q}$. Since $Z$ is compact, $r$ factors through a finite subcomplex $P$ of $Q$ as:

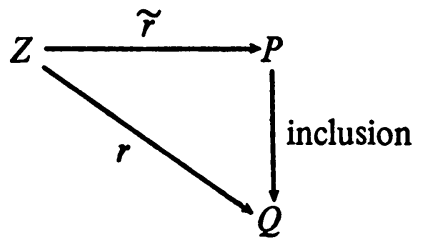

This diagram determines a commutative diagram

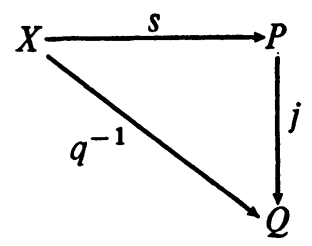

in pro- $H_{0}$ where $s$ corresponds to the homotopy class of $\tilde{r}$ under $\lambda_{P}^{-1}$, and $j$ is generated by inclusion. Thus $q \circ j \circ s=1_{X}$, so $P$ dominates $X$ in pro- $H_{0}$. It follows that $P$ dominates $Q$ in $H_{0}$, and the first part of (iv) is proved. Let $w(Q)$ $\in \widetilde{K}^{0}\left(\pi_{1}(Q)\right)$ be the Wall obstruction of $Q$. Then (see [26, Theorem F]) $w(Q)=$ 0 if $Q$ is homotopy equivalent to a finite complex, and if $w(Q)=0, Q$ is homotopy equivalent to a finite complex of dimension $\max \{3, C W$-dim $Q\}=$ $\max \{3, h-\operatorname{dim} X\}$ by (ii). Define $w(X)=q_{\#}(w(Q)) \in \widetilde{K}^{0}\left(\check{\pi}_{1}(X)\right)$. Since $q$ induces an isomorphism from $\pi_{1}(Q)$ to $\check{\pi}_{1}(X)$ (see (**) and Lemma 4.1) $w(X)=0$ if and only if $w(Q)=0$. Since $w(Q)$ is a homotopy invariant (i.e. any homotopy equivalence $Q_{1} \rightarrow Q_{2}$ carries $w\left(Q_{1}\right)$ to $w\left(Q_{2}\right)$ ) the definition of $w(X)$ is independent of $Q$ and $q$. Finally since $P$ is a subcomplex of $Q, P$ has the required dimension, so (iv) is proved.

PROOF OF (v). Let $\pi$ be a finitely presented group and let $w \in \widetilde{K}^{0}(\pi)$. Following the proof of Theorem $\mathrm{F}$ of [26], one can construct: a pointed $C W$ complex $Q$, a pointed two-dimensional finite $C W$ complex $P$, pointed maps

$$
Q \stackrel{u}{\rightarrow} P \stackrel{d}{\rightarrow} Q
$$

such that $d \circ u$ induces the identity in $H_{0}$, and an isomorphism $\varphi: \pi \rightarrow \pi_{1}(Q)$, such that $\varphi_{\#}(w)=w(Q) \in \widetilde{K}^{0}\left(\pi_{1}(Q)\right)$. Let $f=u \circ d: P \rightarrow P$. Then the tower 


$$
X=\{P \stackrel{f}{\leftarrow} P \stackrel{f}{\leftarrow} P \stackrel{f}{\leftarrow} \cdots\}
$$

in pro- $C W_{0}$ is compact and $C W-\operatorname{dim} X=2 . X$ is isomorphic to $Q$ in pro- $H_{0}$ since $f \circ f$ is homotopic to $f$ in $H_{0}$. To see this( $(2)$ consider the diagram in $H_{0}$ :

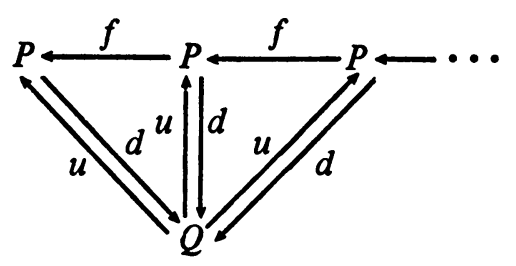

The upward arrows determine a morphism $\bar{u}: Q \rightarrow X$ and the downward arrows determine a morphism $\bar{d}: X \rightarrow Q$ (both morphisms in pro- $H_{0}$ ). Clearly $\bar{d} \circ \bar{u}=1$ and $\bar{u} \circ \bar{d}=1$. Since $w(Q)$ is a homotopy invariant, $w(X)=\bar{u}_{\#}(w(Q))=\bar{u}_{\#} \varphi_{\#}(w) \in$ $\widetilde{K}^{0}\left(\check{\pi}_{1}(X)\right)$.

5. Algebraic criteria for a compactum to be shape equivalent to an ANR and to a compact polyhedron. Let $Z$ be a pointed closed connected subset of some euclidean space $E$. Following Fox [13] we associate with $Z$ the object $F(Z)=\left\{X_{\alpha}\right\}$ in pro-ANR $R_{0}$, where $X_{\alpha}$ ranges over all the connected open neighborhoods of $Z$ in $E$ pointed by the base point of $Z$, and the bonding maps are inclusions. $Z$ has the pointed Fox shape of an object $Y$ of $A N R_{0}$ if and only if $F(Z)$ is isomorphic to $Y$ in pro-HANR $R_{0}$; compare [8]. We define pro- $\pi_{k}(Z)$ to be the pro-group $\left\{\pi_{k}\left(X_{\alpha}\right)\right\}$ and $\pi_{k}(Z)$ to be the inverse limit group $\lim _{\alpha}\left\{\pi_{k}\left(X_{a}\right)\right\}$. Fox proves that when $Z$ is compact his shape theory agrees with that of Borsuk $([13, \S 4]$, the pointed version is identical). It is clear therefore that if $Z$ is compact $\underline{\pi}_{k}(Z)$ is isomorphic to Borsuk's $k$ th shape group (or $k$ th fundamental group) of $Z$ as defined in [2]. $Z$ is movable if for any neighborhood $U$ of $Z$ there exists a neighborhood $V$ of $Z$ with the following property. For any neighborhood $W$ of $Z$ there is a deformation $D: V \times I \rightarrow U$ of $V$ such that $D(V \times\{1\}) \subset W$ and $D$ (base point $\times I)=$ base point. For the definition of pointed fundamental absolute neighborhood retract (pointed FANR) see [3]. The (covering) dimension of $Z$ will be denoted by $\operatorname{dim} Z$.

THEOREM 5.1 (stability theorem for compacta). Let $Z$ be a pointed connected compact subset of some euclidean space.

The following are equivalent:

(i) pro- $\pi_{k}(Z)$ is isomorphic to $\pi_{k}(Z)$ in pro-Groups for each $k \geqslant 1$;

(ii) $Z$ has the pointed Fox shape of a pointed metric simplicial complex whose $C W$ dimension is $\max \{3, \operatorname{dim} Z\}$;

${ }^{2}$ ) We are informed that this trick was previously known to W. Holsztynski. 
(iii) $Z$ is dominated in pointed Borsuk shape by a pointed finite simplicial complex;

(iv) $Z$ is movable and the natural topology on $\underline{\pi}_{k}(Z)$ is discrete for each $k \geqslant 1$;

(v) $Z$ is a pointed FANR.

Furthermore if $Z$ satisfies any of these conditions, there is a "Wall obstruction" $w(Z) \in \widetilde{K}^{0}\left(\pi_{1}(Z)\right)$ which vanishes if and only if $Z$ has the pointed Borsuk shape of a pointed finite simplicial complex. If $w(Z)=0$, the finite complex may be chosen to have dimension $\max \{3, \operatorname{dim} Z\}$. All possible Wall obstructions occur among two-dimensional compacta.

Since $\widetilde{K}^{0}\left(\pi_{1}(Z)\right)$ is trivial if $\underline{\pi}_{1}(Z)$ is free or free abelian (see [26]) we may immediately deduce

Corollary 5.2. Let $Z$ be a pointed connected compact subset of some euclidean space, and let $\underline{\pi}_{1}(Z)$ be free or free abelian. $Z$ has the pointed Borsuk shape of a pointed finite simplicial complex if and only if pro- $\pi_{k}(Z)$ is isomorphic to $\underline{\pi}_{k}(Z)$ in pro-Groups for each $k \geqslant 1$. If this condition holds, the finite complex may be chosen to have dimension $\max \{3, \operatorname{dim} Z\}$.

Proof of 5.1. By triangulating the neighborhoods of $Z$ we may regard $F(Z) \equiv\left\{X_{\alpha}\right\}$ as an object of pro-CW . Since $Z$ is compact, there is a cofinal tower in $C W_{0}, X \equiv\left\{X_{\alpha_{n}}\right\}$.

(i) implies (ii). $\pi_{k}(X)$ is isomorphic to $\check{\pi}_{k}(X)$ for each $k \geqslant 1$. By $4.2, X$ is isomorphic in pro- $H_{0}$ to a $C W$ complex $Q$ of dimension $\max \{3, h-\operatorname{dim} X\}$. Clearly $\operatorname{dim} Z$ is finite. If $\operatorname{dim} Z=d$ there is a tower $X^{\prime}$ in $C W_{0}$, built of nerves of covers of $Z$, such that $C W$-dim $X^{\prime}=d$ and $\lim X^{\prime}$ is homeomorphic to $Z$. By Theorem 12 of [21], $X^{\prime}$ is isomorphic to $X$ in pro- $H_{0}$. Hence $h-\operatorname{dim} X \leqslant d$. Hence $C W$-dim $Q \leqslant \max \{3, \operatorname{dim} Z\}<\infty$. By Lemma 3.11 we may assume that $Q$ is a simplicial complex. Let $Q^{\prime}$ be $Q$ with the metric topology. The "identity": $Q \rightarrow Q^{\prime}$ is a homotopy equivalence $[19, \mathrm{p} .131]$, so $X$ is isomorphic to $Q^{\prime}$ in pro-HT $T_{0}$. But $Q^{\prime}$ is a metric ANR $[17$, p. 106]. Hence $Z$ has the pointed Fox shape of $Q^{\prime}$.

(ii) implies (iii). With notation as above, $X$ is isomorphic in pro- $H_{0}$ to $Q$. Since $X^{\prime}$ may be chosen to be compact, (iii) follows from 4.2(iv).

(iii) implies (iv). $Z$ is movable by 2.3 of [5] $\underline{\pi}_{k}(Z)$ is discrete because lim is a functor from pro-Groups to Topological Groups (see [8]), and $Z$ is dominated by a complex.

(iv) implies (i). By Theorem 2 of [8].

The equivalence of (iii) and (v) follows from [4] and Corollary 6.8 of [3].

Finally, the statements about the Wall obstruction follow from the corresponding statements in 4.2 . 


\section{BIBLIOGRAPHY}

1. M. Artin and B. Mazur, Etale homotopy, Lecture Notes in Math., vol. 100, SpringerVerlag, Berlin and New York, 1969. MR 39 \#6883.

2. K. Borsuk, Concerning homotopy properties of compacta, Fund. Math 62 (1968), 223-254. MR 37 \#4811.

3. - Fundamental retracts and extensions of fundamental sequences, Fund. Math. 64 (1969), 55-85; errata, ibid. 64 (1969), 375. MR 39 \#4841.

4. - On the shape of FANR sets, Bull. Aćad. Polon. Sci. Sér. Sci. Math. Astronom. Phys. 17 (1969), 529-532. MR 41 \#1050.

5. - Some remarks concerning the shape of pointed compacta, Fund. Math. 67 (1970), 221-240. MR 42 \#1076.

6. A. K. Bousfield and D. M. Kan, Homotopy limits, completions and localizations, Lecture Notes in Math., vol. 304, Springer-Verlag, Berlin, 1972.

7. J. Draper and J. Keesling, An example concerning the Whitehead theorem in shape theory (to appear).

8. D. A. Edwards and R. Geoghegan, Compacta weak shape equivalent to ANR's, Fund. Math. (to appear).

9. - Shapes of complexes, ends of manifolds, homotopy limits and the Wall obstruction, Ann. of Math. (2) 101 (1975), 521-535.

10. Infinite-dimensional Whitehead and Vietoris theorems in shape and prohomotopy, Trans. Amer. Math. Soc. (to appear).

11. - Stability theorems in shape and pro-homotopy (to appear).

12. S. Eilenberg and N. Steenrod, Foundations of algebraic topology, Princeton Univ. Press, Princeton, N. J., 1952. MR 14, 398.

13. R. H. Fox, On shape, Fund. Math. 74 (1972), no. 1, 47-71. MR 45 \#5973.

14. R. Geoghegan and R. C. Lacher, Compacta with the shape of finite complexes, Fund. Math. (to appear).

15. D. Handel and J. Segal, An acyclic continuum with non-movable suspension, Bull. Acad. Polon. Sci. Sér. Sci. Math. Astronom. Phys. 21 (1973), 171-172. MR 47 \#5813.

16. P. J. Hilton, Homotopy theory and duality, Gordon and Breach, New York, 1965. MR 33 \#6624.

17. S.-T. Hu, Theory of retracts, Wayne State Univ. Press, Detroit, Mich., 1965. MR 31 \#6202.

18. D. S. Kahn, An example in Čech cohomology, Proc. Amer. Math. Soc. 16 (1965), 584. MR 31 \#4027.

19. A. T. Lundell and S. Weingram, The topology of CW complexes, Van Nostrand Reinhold, New York, 1969.

20. S. Mardešić, On the Whitehead theorem in shape theory, Fund. Math. (to appear).

21. S. Mardešić and J. Segal, Shapes of compacta and ANR-systems, Fund. Math. 72 (1971), 41-59. MR 45 \#7686.

22. J. P. May, Simplicial objects in algebraic topology, Van Nostrand Math. Studies, no. 11, Van Nostrand, Princeton, N. J., 1967. MR 36 \#5942.

23. M. Moszyńska, The Whitehead theorem in the theory of shape, Fund. Math. 80 (1973) $221-263$.

24. D. S. Rim, Modules over finite groups, Ann. of Math. (2) 69 (1959), 700-712. MR $21 \# 3474$.

25. E. Spanier, Algebraic topology, McGraw-Hill, New York, 1966. MR $35 \# 1007$.

26. C. T. C. Wall, Finiteness conditions for CW-complexes, Ann. of Math. (2) 81 (1965), 55-69. MR 30 \#1515. 